\title{
A Framework for Hierarchical Perception- Action Learning Utilizing Fuzzy Reasoning
}

\author{
David Windridge, Michael Felsberg and Affan Shaukat
}

\section{Linköping University Post Print}

\section{Tweet}

N.B.: When citing this work, cite the original article.

(C)2013 IEEE. Personal use of this material is permitted. However, permission to reprint/republish this material for advertising or promotional purposes or for creating new collective works for resale or redistribution to servers or lists, or to reuse any copyrighted component of this work in other works must be obtained from the IEEE.

David Windridge, Michael Felsberg and Affan Shaukat, A Framework for Hierarchical Perception-Action Learning Utilizing Fuzzy Reasoning, 2013, IEEE transactions on systems, man and cybernetics. Part B. Cybernetics, (43), 1, 155-169.

http://dx.doi.org/10.1109/TSMCB.2012.2202109

Postprint available at: Linköping University Electronic Press

http://urn.kb.se/resolve?urn=urn:nbn:se:liu:diva-85688 


\title{
A Framework for Hierarchical Perception-Action Learning Utilizing Fuzzy Reasoning
}

\author{
David Windridge, Michael Felsberg, and Affan Shaukat
}

\begin{abstract}
Perception-Action (P-A) learning is an approach to cognitive system building that seeks to reduce the complexity associated with conventional environment-representation/actionplanning approaches. Instead, actions are directly mapped onto the perceptual transitions that they bring about, eliminating the need for intermediate representation and significantly reducing training requirements. We here set out a very general learning framework for cognitive systems in which online learning of the P-A mapping may be conducted within a symbolic processing context, so that complex contextual reasoning can influence the $P$ A mapping. In utilizing a variational calculus approach to define a suitable objective function, the P-A mapping can be treated as an on-line learning problem via gradient descent using partial derivatives. Our central theoretical result is to demonstrate topdown modulation of low-level perceptual confidences via the Jacobian of the higher levels of a subsumptive P-A hierarchy. Thus, the separation of the Jacobian as a multiplying factor between levels within the objective function naturally enables the integration of abstract symbolic manipulation in the form of fuzzy deductive logic into the P-A mapping learning. We experimentally demonstrate that the resulting framework achieves significantly better accuracy than using perception-action learning without top-down modulation. We also demonstrate that it permits novel forms of context-dependant multi-level P-A mapping, applying the mechanism in the context of an intelligent driver assistance system.
\end{abstract}

Index Terms-Perception-Action Learning, Online Learning, Fuzzy Logic, Subsumption Architectures, Machine learning, Hierarchical systems, Autonomous agents, Vehicle safety.

\section{INTRODUCTION}

\section{A. Objective}

$\mathbf{T}$ HE object of this paper is to propose a novel approach to building multi-stage perception-action (P-A) systems. Layered systems have been state of the art since the early 1980s, e.g., in terms of the subsumption architecture [1]. However, where the latter was focused on finite state machines, the alternative proposed here is that of a layered system with explicit P-A inputs and outputs at each stage of the system. The result is thus an embodied system that uses the 'actionprecedes-perception paradigm' [2]. More recently, this concept

Manuscript received August 9, 2011; revised January 30, 2012 and May 1, 2012; accepted May 4, 2012. Date of publication May 12, 2012; date of current version Sep 15, 2012. This paper was recommended by Associate Editor XYZ.

D. Windridge and A. Shaukat are with the Centre for Vision, Speech and Signal Processing, FEPS, University of Surrey, Guildford, UK (e-mail: d.windridge@surrey.ac.uk; a.shaukat@ surrey.ac.uk).

M. Felsberg is with the Department of Electrical Engineering, Linköping University, S-58183 Linköping, Sweden (e-mail: mfe@ isy.liu.se).

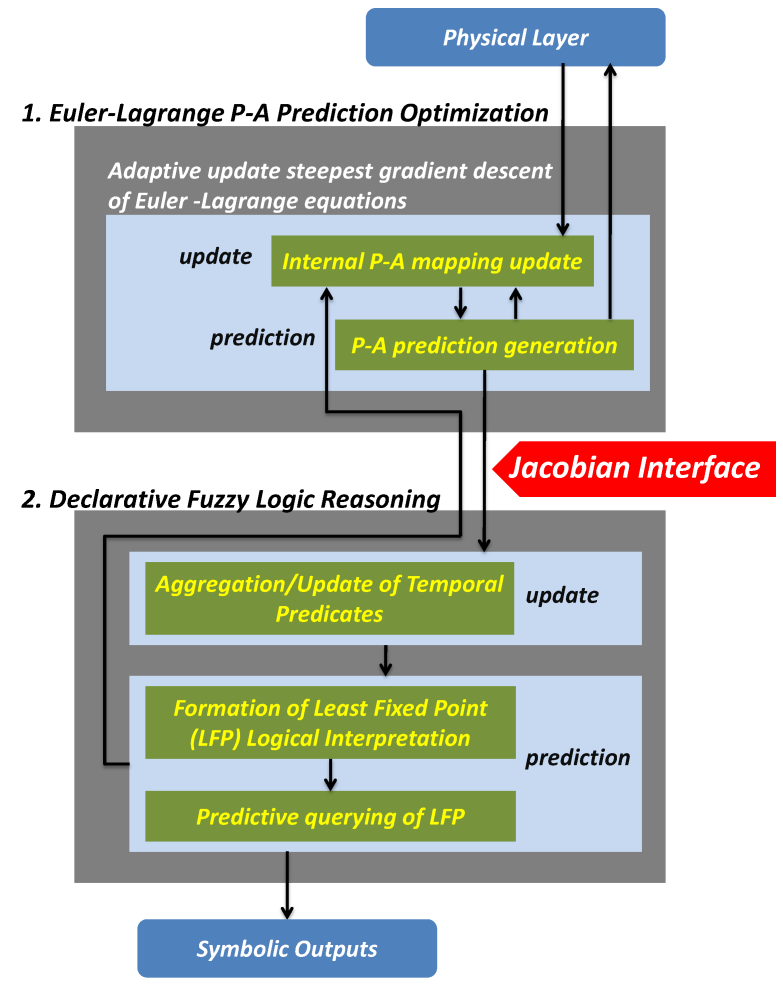

Fig. 1. Algorithmic process flow diagram for the integrated P-A learning framework.

has been extended to continuous learning employing exploration and adaptation [3]. In the present paper, we describe these notions in terms of concepts defined in [4].

Implicit in the above, our aim is to set out a general learning framework for cognitive systems in which online learning of the P-A mapping may be conducted within a symbolic processing context, so that complex contextual reasoning can influence the P-A mapping. In utilizing a variational calculus approach to define a suitable objective function, the P-A mapping can be treated as an on-line learning problem via gradient descent using the Euler-Lagrange Equation. Our central theoretical result is to demonstrate top-down modulation of low-level perceptual confidences via the Jacobian of the higher levels of a subsumptive P-A hierarchy. Thus, the separation of the Jacobian as a multiplying factor between levels within the objective function naturally enables the integration of abstract symbolic manipulation in the form of fuzzy deductive logic into the P-A mapping learning. In terms of algorithmic process flow, the integrated system is as shown in Fig. 1. 
We experimentally demonstrate that the resulting framework achieves significantly better accuracy than using perceptionaction learning without top-down modulation. It further permits novel forms of context-dependant multi-level P-A mapping and learning, which we illustrate qualitatively for two distinct experimental scenarios in the context of intelligent driver assistance systems:

1) Logical closure of sparse/erroneous detector predication (i.e., the on-line formation of a maximally logically selfconsistent model of actions and perceptions).

2) Adaptive appearance-label/semantic-logic association (via the the on-line attachment of detector labels to their corresponding functional meaning).

The scenarios involve both top-down and bottom-up constraints on the learning system, illustrating the bi-directionality of the multilevel online P-A learning. The system as a whole can thus, in principle, perform several iterations of top-down and bottom-up adaptation to enact a 'cognitive bootstrap' process (along the lines of [5]), in which novel perception-action hypotheses are constructed and projected into the environment for consistency testing.

\section{B. Perception-Action Systems}

We define our notion of perception and action, as well as other key terms, more formally as follows.

Percepts are virtual representations of observable physical system states. These states are called visible states in [4].

Actions are changes of physical system states initiated by virtual states.

Virtual system states are states of the system, which do not have a physical realization - except for their physical implementation in terms of memory cells - e.g., variables. These states are hidden states, internal to the estimator in [4]. They may, very loosely, be considered 'mental' states.

Physical system states are states of the system that have a physical realization, e.g., angles of a robotic arm.

State mappings are mappings taking percepts and virtual system states as input and delivering virtual system states and actions as output. These mappings are called inverse mappings in [4].

A cognitive system is a system consisting of all previous items, where at least a part of the mappings is learnable and adaptable.

Note that percepts and actions are always with respect to system states, i.e., the interface between physical entities and virtual entities lies within the system. The system interacts with the world through its physical states, i.e., the interface to the world is realized by physical interaction of states, e.g., mechanical laws or transmission of light.

The underlying paradigm behind P-A learning is that " $a c$ tion precedes-perception learning" [2], implying that perceptual states should not be presumed to exist in the absence of actions capable of distinguishing them (assuming an embodied agent). This typically has the benefit of reducing the size of a percept space by several order of magnitude (particularly when dealing with vision-based systems) - Action spaces, on the other hand, usually employ only a few degrees of freedom and have low intrinsic dimensionalities. The aim of a practical PA system is thus to establish (preferably bijective) mappings from percepts to actions. For this purpose, both spaces need to be structured. This can be approached in supervised and unsupervised ways. In cognitive bootstrapping [6] the system faces entirely unknown P-A mappings and establishes them by performing random actions, greedily labeling the corresponding percepts, and then generalizing the resulting perceptual domain so as to suggest novel action possibilities. (This latter aspect can be performed off-line). During the exploration phase, mappings are tested by performing actions

In the current paper, we are rather dealing with a supervised system, in which the actions are those of a car driver, and the percepts are the (computer vision-based) detections of significant objects within the driver's field of view. Thus, we are, in effect, modelling the human P-A system used in driving, so as produce a system ultimately capable of driver assistance (although beyond the scope of this paper).

\section{Symbolic Processing Within P-A Learning Systems}

The driving environment adds additional considerations into standard P-A learning, namely those of high-level context and protocol-driven events. It also involves an explicit, pre-existing symbol domain (signs, traffic-lights, etc). We must therefore address both the classical problem of symbol grounding [7], but also that of contextual association.

It is clear from the the nature of the protocols governing road-use that to exploit context in the most generally flexible way, we must employ logical deduction. However, we are then faced with the problem of how to integrate stochastic information with logic; the classical problem of symbolic/subsymbolic integration [8], [9].

To date, the problem of optimal perception-action mapping has thus not been considered in the context of a subsumption architecture that integrates logical processing for contextual reasoning. The aim of this paper is to demonstrate how fuzzy first-order resolution can be directly incorporated into variational calculus so as to optimize the P-A mapping of an embedded multi-level cognitive system. In doing so, we hope to incorporate a capacity for generalizing beyond the immediate context, and thereby bring about faster overall convergence on the optimal P-A mapping. We therefore build a hybrid adaptive P-A learning system that, uniquely, employs top-down feedback for improving convergence and extending capabilities in a number of unique ways.

Moreover, in applying P-A learning in a first-order logical context, we go beyond the classical problem of symbolic and sub-symbolic integration, extending it to that of symbol tethering [10], [11].

\section{Details of the Application Domain}

To demonstrate the application of the deductive P-A learning system, we apply it within the context of an Instrumented Vehicle that incorporates driver gaze tracking, a forward camera, control input measurements, and also LIDAR range finding (and also more driver-physiology based inputs: skin resistivity, 'a brake hover' camera, etc). The vehicle is 


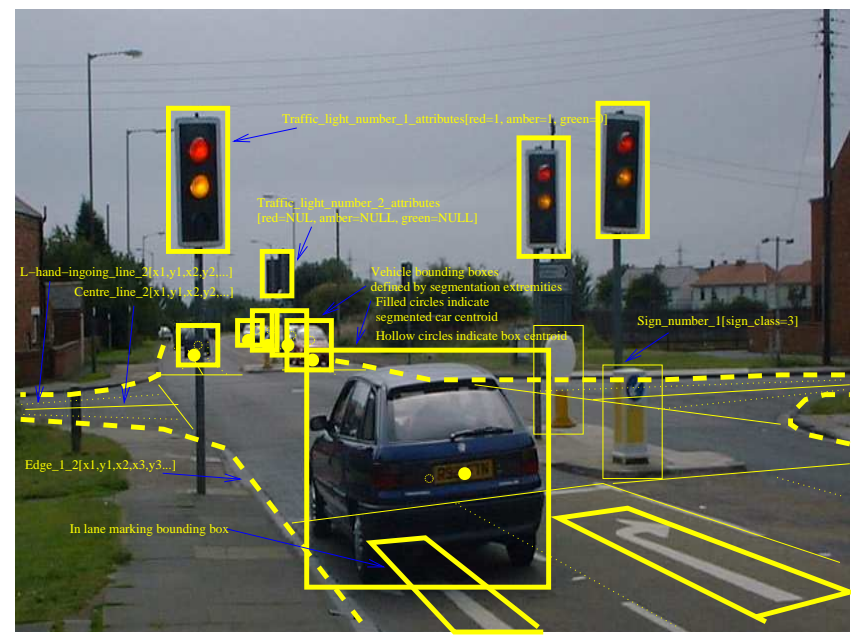

Fig. 2. Ideally-complete set of highway-code-relevant detector outputs within camera frame.

thus equipped with a non-intrusive four camera eye tracking system that continuously records the driver's gaze direction. Three roof-mounted cameras with a 180 degree field of view capture the environment and traffic dynamics; the driver's gaze direction can be overlaid onto the video from the roofmounted cameras. Cameras and proximity detectors in the foot well and relevant data on the CAN bus can be recorded to further capture the driver's activities. Navigation is done with differential GPS and fibre optic gyroscope. This vehicle is utilized in both normal city driving contexts as well as a Specialist Test Track with simulated pedestrians, cars etc.

The forward Camera feed is interpreted via a Computer Vision System incorporating a range of visual detectors. An idealized set of visual detectors (with associated bounding boxes for gaze location), is depicted in Fig. 2; it consists of perfect car, lane sign and light detectors. With such an idealized set of detectors, it is possible, in principle, to make an optimal deduction as to the likely outcome of any traffic situation (i.e., the detectors are completely informative with respect to the legal protocols of the driving situation).

However, in practice, due to the inherent limitations of instrumental detection, only a subset of these protocol-salient detections may be present at any given time.

The task of the autonomous cognitive system outlined in this paper when applied to the driving environment is thus to make predictions as to driver behavior with respect to the external scene; these will then constitute the Jacobian with which the separate P-A modules are interfaced.

\section{E. Use of symbol processing within an adaptive P-A model of driver behavior}

From an externalized perspective, the central mode of operation of the system is to infer driver's intentions with respect to a complete model of the external scene (as sparsely measured by the pattern-recognition and computer-vision based detectors). Such a system might form the basis for cognitive driver assistance system, capable of issuing warnings.
For this we need an a priori model of intentionality that may be applied to the objects of the road. We hence use the psychologically-motivated Extended Control Model (ECOM) model [12] as a basis for interfacing Highway Code protocols with the Perception-Action hierarchy.

The ECOM model, while originally developed as a conceptual P-A model, lends itself to clausal description in terms of key objects of observation. These clauses can be combined with HighWay Code Protocols to form a Logical Rule Base (i.e., a set of clauses) appropriate to a driver assistance system.

From an internal perspective, the aim of the system is thus to adaptively reconcile computer-vision/control/gaze inputs with the known behavioral protocols (i.e., in our experimental domain, the UK Highway Code and ECOM models). The problem is thus one of the combination of abstract deduction with pre-symbolic input. In this way we address the wider cognitive science issues of symbol grounding [7] and symbol tethering (according to Sloman's usage [11]).

\section{F. Structure of Paper}

We first (section II) give a modular outline of the proposed stacked P-A learning system, and show generically how learning is propagated. In section III we indicate the requirement for symbolic reasoning to be incorporated into this structure. Section IV sets out the argument for Fuzzy Deductive Logic as having the requisite methodological characteristics for incorporation into the stacked P-A learning system. In section $\mathrm{V}$ we show how online layer-adaptation can be carried out this framework using an Euler-Lagrange approach. We demonstrate, in particular, the multiplicative separability of feedback factors within the interlayer Jacobian. Section V concludes with a worked derivation using the example of a protocol-driven driver assistance system, and demonstrates algorithmically how the multiplicative factor arises in practice.

We then, in section VI-A, utilize this context to demonstrate quantitatively that feedback improves lower-level learning in isolation. After demonstrating quantitative evidence of improved learning through a suitable multiplicative factor, we give, in Section VI-B, a demonstration of how this multiplicative factor arises in a real online scenario, enabling complex behavior such as adaptive symbol tethering. We conclude in Section VII with a summary of achievements.

\section{A Learning Framework For Multi-Layered P-A HIERARCHIES}

The major theoretical undertaking of this paper is thus to present an approach to Multi-Level P-A Learning, one which will perform optimal P-A mapping utilizing feedback/control from higher levels (the resulting method can be applied recursively for any number of levels). For this we use a Variational Euler-Lagrange-based approach for system integration.

The major novelty of the theoretical component of the paper is thus the integration of Fuzzy Logic Theorem Proving into such a variational approach to accommodate uncertainty of detector input (supplied as predicates). Our approach is thus one of symbolic/sub-symbolic integration in which both levels are instances of the same structure. 


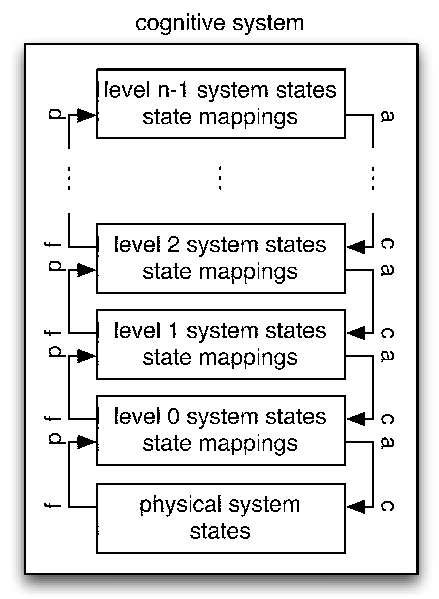

Fig. 3. Layered system architecture and related terminology: percepts (p), actions (a), feedback (f), and control (c).

\section{A. A Multi-Layered P-A System: terminological definitions}

It is thus possible to extend the learning-based P-A system structure suggested in [3] to several layers in order to form a subsumptive hierarchy. This extension is required as adaptation requires top-down control. (The presented ideas show large overlap with the structure presented in [13] with the main difference that the cited work only considers perception).

Some addition terminology is required to extend the notion of percepts and actions to higher levels, in particular the terms control (a higher level action) and feedback (a higher level percept) are introduced:

Percepts are virtual representations at level $n$ of observable system states at level $n-1$ if $n>0$ or virtual representations of observable physical system states at level 0. Actions are changes of system states at level $n-1$ initiated by virtual states at level $n$ if $n>0$ or physical system states initiated by virtual states at level 0 . Feedback is the output of level $n$ that is considered as percepts at level $n+1$ and control is the input to level $n$ that is considered as actions at level $n+1$.

These concepts allow us to build a system with several layers of virtual states and mappings, as illustrated in Fig. 3. Note that level 0 can be considered as an innate (engineered) level and $n$ grows with time.

Note also the similarity to the original subsumption architecture [1]; however the latter uses finite state machines.

It will generally be the case that, in forming a Subsumptive hierarchy we obtain progressively generalized percepts and progressively generalized actions. Taking this idea to its extreme, the highest level of the hierarchy should be capable of abstract symbol manipulation. The most general framework for such symbol manipulation applicable to general system scenarios would thus be First Order Logical deduction.

\section{B. Learning in Multi-Layered Systems}

The considered system does not have distinct modes for learning and working. From the beginning, it has no capabilities except for level 0 . This level can be considered as a (virtual representation of) physical state predictor. Whenever the prediction fails, a feedback is triggered to level 1, indicating a need for changing the control of level 0 , i.e., requesting an action at level 1. In this way, level 1 is embedded in a percept-action cycle and acts as a predictor for level 0 states. This leads to bootstrapping of level 1, using the mechanisms described earlier. When the level 1 predictions start to work, bootstrapping of level 1 can be considered completed.

The feedback of level 1 (being sent to level 2), becomes stronger with time and eventually triggers a similar bootstrapping process at level 2. Meanwhile, level 1 still adapts, continuously trying to improve its predictions. By sending different control signals from level 2 to level 1 , level 1 develops different modes of prediction, i.e., it starts to make different predictions based on the top-down control. If the feedback from level 0 (the percept of level 1) becomes stronger than a triggering threshold, level 1 will generate a new mode of prediction, thus exploratory learning is applied as described earlier.

Repeating this procedure through more and more levels leads to a system with increasingly better capabilities w.r.t. solving a task of appropriate complexity. It is important to choose the complexity such that exactly one new level needs to be generated in each step, otherwise learning might become slow or entirely break down, very similar to learning in humans.

We note here the similarity of what is being proposed to PAC modules [14]. 'P' stands for percept, 'A' for action, and ' $\mathrm{C}$ ' for control. By extending P-A learning with a top-down control, P-A functionalities go beyond homeostatic behavior. In a predictive coding or tracking approach to perception, $\mathrm{P}$-A functionality is achieved by prediction [4]. Extending predictive coding by top-down control means to modulate prediction functionalities, or, as known in the tracking literature, to switch models [15]. In PACs, the feedback to the respectively higher module is part of the action output. However, the downwards output from a PAC module (the action) might have a different time-scale and representation as the upwards output (feedback). Thus we propose to extend PACs with an explicit feedback channel $\mathrm{F}$, resulting in PACFs.

Similar to the PACs, the PACFs are related to the Neisser perceptual cycle [16] and can be interpreted as an abstract tracking process that is looping through the reflexive transitive closure of (state - system model - prediction - matches - observation - update). The matching of predictions and observations (percepts) defines the internal modelling error and drives adaptation and feedback. Small errors lead to adaptation, whereas large errors lead to feedback and exploration or bootstrapping at the next higher level. The proposed learning structure has been evaluated in a single layer system using off-line learning in [17].

\section{The Symbol Processing LAyer}

In the system we have outlined, the symbol processing layer will be the most abstract (i.e., topmost) level of the P-A hierarchy. Actions at this level cascade down the PA hierarchy, acquiring more and more low-level context. To achieve maximal generality for abstract reasoning in practical 
domains this level should therefore incorporate First Order Logical deduction (i.e., reasoning with quantifiers and predicates), so that novel and hypothetical symbol combinations can be considered.

However, detections from the low level detectors are typically passed to the symbol processing layer along with associated confidences (in some cases these will be derived stochastically from validation data, and in some cases this will be a more ad hoc certainties based on prior knowledge). The role of the feedback process from the symbol processing layer in this case is thus to re-weight confidences in an appropriate manner. We must therefore perform first order logical deduction in an uncertain environment.

In general, there are two distinct ways of dealing with stochastic uncertainty in logic: Markov Logic Networks (MLNs) [18] [19] [20] and Fuzzy Logic (FL) [21] [22]. MLNs deal with uncertain clauses (i.e., rules) and FL with uncertain predication. In many domains in which we might wish to deploy an artificial cognitive system, including our domain, we have relatively certain rules (the Highway Code, the ECOM model, the laws of physics) in combination with uncertain detector predication (and often also non-strictly stochastic detector confidences). These factors therefore suggest that we use Declarative Fuzzy Logic rather than MLNs for the topmost symbol processing layer in our P-A hierarchy.

Note that declarative Fuzzy Logic allows much more general deductive reasoning than standard non-recursive FL control applications, which are commonly encountered in control theory (see e.g., [23] [24]). We require a system capable of querying of hypotheticals and theorem proving in order to fully explore the influence of context.

\section{Declarative Fuzzy Logic Programming}

\section{A. Fuzzy Theorem Proving}

Fuzzy logic is the logic applicable to fuzzy sets, i.e., sets for which there are degrees of membership. This is usually formulated in terms of a membership function valued in the real unit interval $[0,1]$. Various fuzzy logics are possible within this framework; membership functions (and therefore truth values) can be single values, intervals or even sets of intervals within the unit interval $[0,1]$. While not strictly stochastic, fuzzy logic programming is well suited to the implementation of methodologies relating to reasoning with uncertainty [25].

The propagation of truth values through logic rules is carried by means of operators. This subsumes conjunctive operators (T-norms; min, prod etc) and disjunctive operators (T-conorms; max, sum, etc) as well as hybrid operators (combinations of the previous operators) [26]. In particular, the T-norm (triangular norm) is generally a continuous function, $[0,1] \times[0,1] \Rightarrow$ $[0,1]$, equivalent to classical conjunction for the integer truth values 0 and $1[27]^{1}$.

The implication operator can consequently be constructed as a residuum of the T-norm. Thus there is a unique binary operation $\Rightarrow$ on $[0,1]$ such that:

$$
(x \Rightarrow y)=\sup \{\gamma \mid T(\gamma, x) \leq y\}
$$

thus, $\forall(x, y, \gamma) \in[0,1]$,

$$
(x \Rightarrow y)=1 \quad \text { iff } \quad x \leq y \quad \text { and } \quad(1 \Rightarrow y)=y
$$

Fuzzy logic thus allows for the representation of clauses as the implication of a head predicate with respect to a conjunct of body literals. Modus ponens arguments can thus be formalized within FL; however the rule of resolution is not strictly axiomatizable in multi-valued logic without either additional structure to the truth values, or else limitations on the permissible depth of resolution.

This ability to represent clauses in uncertain environments has lead to the widespread use of FL within control applications [31] [32]. However, perhaps because of the above issues, what is generally not considered in such applications is declarative fuzzy logic, such that arbitrary queries can be addressed to the logic system. This sort of flexibility, however, is precisely what is required in a domain such as cognitive driver assistance, where generalized rules may lead to highly complex context-dependant conditionalities. Neither compositional fuzzy reasoning nor fuzzy rule interpolation [33] allow for resolution theorem-proving i.e., first-order logical deduction with instantiation, such as is required to deduce possible outcomes of particular traffic configurations (such approaches do not perform 'symbolic manipulation' in any cognitive sense).

In the following, we implement our rule system within Ciao Prolog [26], which extends standard ISO-Prolog by incorporating constraint logic programming, $C L P(R)$, [34] to accommodate fuzzy-valued logic. (A number of other approaches to declarative querying with fuzzy logic are possible, but generally these modify the resolution process in fundamental ways, making them potentially more difficult to debug for large rule-bases).

In implementing full first order logical resolution via CLP(R) in CIAO Prolog, we thus differentiate our method from compositional fuzzy reasoning, where fuzzy inference takes place by assigning membership values to output variables with pre-defined rules and membership functions, thereby permitting "Proof-by-refutation" (recursive querying of clauses and instantiation of variables with new values using aggregator operators). Such aggregation schemes subsume conjunction (e.g., via T-norms) as well as disjunction (via e.g., t-conorms) in a way that naturally also leads to truth values that constitute a union over interval subsets of $[0,1]$.

\footnotetext{
${ }^{1}$ T-norms can be continuous or discontinuous. For each discontinuity point in a discontinuous T-norm function, either the left or right limit can be selected as the value of the point (since T-norms have implicit monotonicity). More common in the literature, however, are the left-continuous T-norms e.g., [28] and [29]. Contrarily, the drastic T-norm, the point-wise smallest Archimedian T-norm [30], is an example of a right-continuous T-norm. In the following we use the product T-norm as the generic aggregator (logical connective) operator for the CIAO Prolog based SP module (which is implicitly continuous); discontinuous T-norms fall outside the scope of the paper.
} 
The truth values of a predicate in Ciao Fuzzy Prolog are thus elements of a Borel Algebra over the interval $[0,1]$. The set of continuous subintervals on $[0,1]$ is denoted by $\varepsilon([0,1])$. Thus Borel Algebra, $\mathcal{B}([0,1])$, can be considered as the power set of $\varepsilon([0,1])[35]$. The aforementioned Aggregation operators are used to propagate truth values by means of the fuzzy logic rules. Aggregation in Fuzzy Sets is carried via the application of an aggregation operator of the type: $f:[0,1]^{n} \rightarrow[0,1]$. (In addition to this, it has to satisfy $f(0, \ldots, 0)=0$ and $f(1, \ldots, 1)=1$ and be monotonic).

In Ciao Prolog, given an aggregation $f:[0,1]^{n} \rightarrow[0,1]$, an interval-aggregation $F: \varepsilon([0,1])^{n} \rightarrow \varepsilon([0,1])$ is defined as follows [26]:

$$
F\left(\left[x_{1}^{p}, x_{1}^{q}\right], \ldots,\left[x_{n}^{p}, x_{n}^{q}\right]\right)=\left[f\left(x_{1}^{p}, \ldots, x_{n}^{p}\right), f\left(x_{1}^{q}, \ldots, x_{n}^{q}\right)\right] .
$$

Extending this, given an interval-aggregation $F$ : $\varepsilon([0,1])^{n} \rightarrow \varepsilon([0,1])$ defined over intervals, a unionaggregation $F^{\prime}: \mathcal{B}([0,1])^{n} \rightarrow \mathcal{B}([0,1])$ is defined over union of intervals as follows [26]:

$$
F^{\prime}\left(B_{1}, \ldots, B_{n}\right)=\cup\left\{F\left(\varepsilon_{1}, \ldots, \varepsilon_{n}\right) \mid \varepsilon_{j} \in B_{j}\right\} .
$$

Borel sets thus arise naturally from interval aggregation. Importantly, this Borel set approach to fuzzy logic permits ambiguity in the allocation of fuzzy values without necessitating a higher-order concept of fuzziness. Thus, what would otherwise have to be treated via modification of the resolution procedure to accommodate multiple alternatives (i.e., if using a standard, single-valued fuzzy logic implementation), can instead be accommodated within a single act of fuzzy resolution. It is this that allows fuzzy logic to be directly grafted onto the structures of Prolog.

However, we shall implement a constrained form of $C L P(R)$ in which clausal conjunction is formed via the product T-norm of atomic predicates, with the T-norm operation, max, being applied to the predicates individually. This has the effect of 'de-Borelizing' the detector predication, while retaining the multiplicity of truth values associated with head predicates in the clauses. Borel set truth values thus only occur as a result of the resolution procedure, but do not arise within clauses themselves. This prevents the upper bound of the truth value dominating as resolution progresses. Only at the end of deductive resolution is this resolution-based multiplicity resolved (via the max T-norm). The output is then a single interval truth value for each predicate. In this way we obtain a singular world model in which fuzzy values function like predicate confidences.

\section{B. Fuzzy Prolog Syntax}

Given that $A$ is an atom then a fuzzy fact can be represented as $A \leftarrow v$, where as $v$ is a truth value, an element in $\mathcal{B}([0,1])$, defined as constraints over the domain $[0,1]$.

If $A, X_{1}, \ldots, X_{n}$ are atoms, $A \leftarrow_{F} X_{1}, \ldots, X_{n}$ represents a fuzzy clause where $F$ is an union-aggregation operator of truth values in $\mathcal{B}([0,1])$ defined as constraints over the domain $[0,1]$.
If $A$ is an atom, and $v$ is a variable representing a truth value in $\mathcal{B}([0,1])$, then a fuzzy query is represented as $v \leftarrow A$ ?. A fuzzy program is a collection of facts and rules (i.e., a subset of the Herbrand base mapped into $\mathcal{B}([0,1])$ along with a set of clauses, but excluding queries).

In operational terms, at any given time the state of the fuzzy logic system is described via the tuple: $\langle A, \sigma, S\rangle$ [26]

where $A$ is the goal/query, $\sigma$ is a substitution/instantiation of variables, and $S$ is a 'constraint store' holding truth values of the query at that state.

A single iteration of the resolution algorithm thus executes the transition:

$$
\langle A, \sigma, S\rangle \longrightarrow\left\langle A^{\prime}, \sigma^{\prime}, S^{\prime}\right\rangle \text {. }
$$

Two types of transition, in particular, are possible in attempting to solve query $A$ :

1. The application of facts:

$$
\langle A \cup r, \sigma, S\rangle \longrightarrow\left\langle A \theta, \sigma \cdot \theta, S \wedge \mu_{r}=v\right\rangle
$$

where $q \leftarrow v$ is a fact of the program $P$, and $r$ is a component of the goal $A, \theta$ is the most general unifier of $q$ and $r$, and $\mu_{r}$ is the truth variable for $r$.

2. The application of rules:

$$
\langle A \cup r, \sigma, S\rangle \longrightarrow\langle(A \cup B) \theta, \sigma \cdot \theta, S \wedge c\rangle
$$

where $q \leftarrow{ }_{F} B$ is a rule of the program $P, \theta$ is the 'most general unifier' of $q$ and $r, c$ is the constraint that defines a truth value obtained via the application of the aggregator $F$ to the truth variables of $B$ (if none of the above are applicable then the transition fails).

Resolution comprises a sequence of transitions, initialized with $A$ as the query and $\sigma=\varnothing$ and $S$ set to true; transitions halt when $A$ is empty: $\sigma$ and $S$ represent the final result.

Semantic Meaning within the above system is related to the least model of a program; the concept of 'least' being defined under set inclusion with regard to subsets of the Herbrand base and under lattice inclusion with respect to fuzzy values within the Borel set $\mathcal{B}([0,1])$. Thus, following [26], we define semantic meaning as follows. Firstly, suppose $T_{P}$ is a one-step logical consequence operator (as defined above) for the fuzzy program, $P$ that acts on the model $I$ such that $T_{P}(I)=I^{\prime}$ with $I^{\prime}=<B_{I^{\prime}}, V_{I^{\prime}}>$, where we have that $B_{I}=\left\{B \in B_{P}\right\}$ and $V_{I}(B)=\bigcup\{v \in \mathcal{B}([0,1])\}$ (i.e., $B_{I}$ is a subset of the Herbrand base, and $V_{I}$ is a corresponding allocation of truth values (with zeros otherwise under the default closed world assumption).

The meaning of a fuzzy program, $P$, is then its least fixed point, $\operatorname{lf} p\left(T_{P}\right)=I,\left(T_{P}(I)=I\right)$, with respect to the allocation of fuzzy values to predicates from its Herbrand base, where $I$ is considered an interpretation. We thus equate the notion of a least model, $I$, with stability under the operation of resolution, such that all facts within $I$ are consistent with each other, being thus either axiomatic or provable from axioms. This notion will become important later when we subsume fuzzy Prolog within a hierarchical Euler-Lagrange framework, for which $I$ will constitute a set of internal or virtual system state representing the logical completion of the sparse fuzzy input 'perceptual' input $p$ (i.e., we query over all atoms 
relating to possible perceptual input, such that the complete set of instantiated variables and associated truth values in the program $P$ thus constitute the world model). The least model is thus the optimally self-consistent world model on the basis of an Occam's-razor-like closed world assumption. This means that a self-consistent world model incorporating the sparse perceptual facts and highway-code/ECOM rules can be created via exhaustive querying of the input predicates.

In the following, we shall set out to show how the least fixed-point $I$ (constituting the logic-level virtual road model $V_{t}^{l o g}$ existing at time $t$ in our application domain), may be related, in an online fashion, to the logic-level virtual system state, $r_{t}^{\log }$ used in the second level of the PACF.

\section{The Euler-Lagrange Symbolic/Sub-Symbolic PERCEPTION-ACTION INTERFACE}

\section{A. Adaption of a Layer}

Let us define the following terms: $p_{t}$ is the percept at time $t$ ( $I$ in [4]), $r_{t}$ is the internal state at time $t, a_{t}$ is the action at time $t, c_{t}$ is the control at time $t, f_{t}$ is the feedback at time $t$, and $\tilde{p}_{t}$ is the expected percept a time $t$. Furthermore, we define two mappings $u$ and $v$. The mapping $v$ is the internal state update

$$
r_{t+1}=v\left(c_{t}, \rho\left(\tilde{p}_{t}-p_{t}\right), r_{t}\right)
$$

where $\rho$ is the prediction error function, which typically saturates for large arguments. The internal state is thus updated depending on the top-down control, the internal state, and the prediction error. Usually, a small prediction error will lead to a small update $\left(r_{t+1} \approx r_{t}\right)$, if not, the control signal requests a change. The mapping $u$ is the prediction generation

$$
\tilde{p}_{t}=u\left(r_{t}\right),
$$

which produces an expected percept depending on the internal state. Thus, the internal state models the process generating incoming percepts: the sensor inputs (on the lowest level) or the output from the lower level PACFs. A subset of the prediction is fed back down as a control signal to the next lower level and might influence the processing of the lower levels (adaptive processing). A subset of the internal state is sent up as a feedback signal, representing an abstraction of the incoming information.

The feedback as part of $r(t)$ influences the state vector $r_{t+1}$ and leads to internal adaption through (7). Furthermore, it might lead to changes in the control signal for a future time step $\left(c_{t+t_{c}}\right)$. Actions might lead to new percepts with unknown delay $p_{t+t_{p}}$. A scheme for two interacting PACFs for grounding and logic processing is illustrated in Fig. 4.

The goal of each layer is to achieve a low prediction error $\rho\left(\tilde{p}_{t}-p_{t}\right)$ and at the same time to update the internal state as little as possible. The first criterion is obvious: if the prediction error is low, the system provides good predictions of percepts caused by its actions, i.e., the system shows a high degree of flexibility to model percept-action loops. The second point might be less obvious, but has partly been discussed in [4] and can be further motivated by minimizing efforts to change the system and by implicitly requiring the control to be smooth.

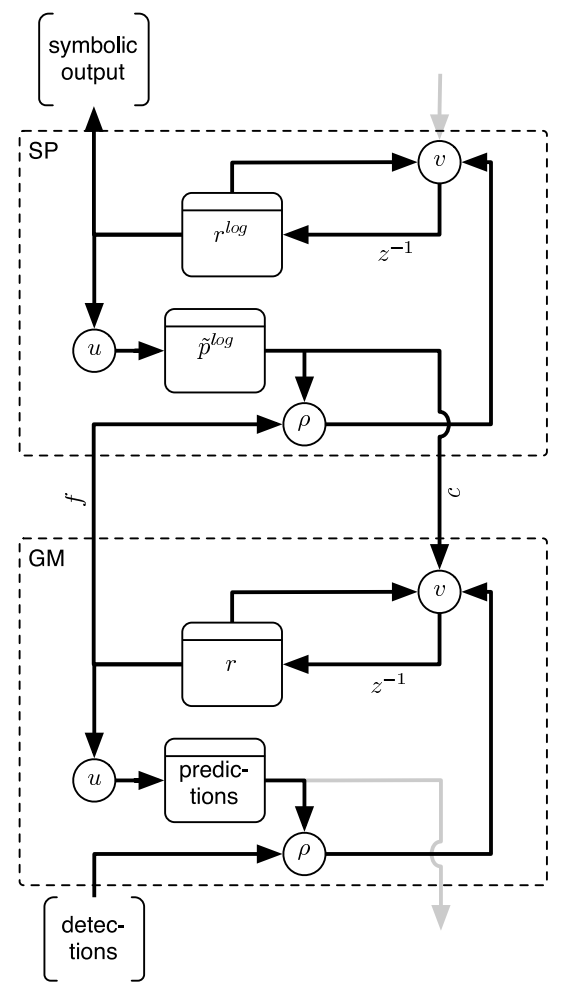

Fig. 4. State diagram of two interacting PACFs GM (grounding module) and SP (symbolic/logical processing) with the internal states $r$ and $r^{\log }$ and the percepts $p=$ detections and $p^{l o g}=f$ (the feedback from GM). The prediction are $\tilde{p}=$ predictions and $\tilde{p}^{l o g}$. Furthermore, the SP controls through $a^{l o g}=c$ the GM, but there is no action $a$ of the GM. Also, there is no control of the SP $c^{\log }$. The feedback of SP is $f^{\log }=$ symbolic output. Finally, $z^{-1}$ denotes a unit time delay.

One implication of the action-precedes-perception paradigm is that actions are continuous for consistent trajectories through percept space. However, percept space is discontinuous and therefore it is easier to measure the consistency of percept sequences by continuity of actions. Therefore, we consider fewer changes of the control signals as an indicator for correct feedback. This corresponds to the generalization capability of the layer under consideration.

\section{B. An Implementation of Learning}

One possibility to formulate the learning problem that we face is in terms of an objective function that is to be minimized. Obviously, we would like to have a good prediction independently of what the control $c_{t}$ and the percept $p_{t}$ are (fidelity). Furthermore, small changes in the percepts should result in small changes of the internal state $r$. These requirements result in

$$
\varepsilon(u, v)=\int \Psi\left(\left|\nabla_{p} r_{t+1}\right|\right)+\rho\left(\tilde{p}_{t}-p_{t}\right) d c_{t} d p_{t}
$$

where $\Psi$ is some error norm applied to the gradient of $r$, e.g., the L1-norm (which will basically count the occurrences of changes of $r$ ). This term acts as a regularization term that punishes too frequent changes of the internal state (essentially a "laziness" term). The term with $\rho$ is the prediction error function, already in the previous section. 
In order to optimize this equation, we substitute in (7) and (8)

$$
\begin{aligned}
\varepsilon(u, v)= & \int \Psi\left(\left|\nabla_{p} v\left(c_{t}, \rho\left(u\left(r_{t}\right)-p_{t}\right), r_{t}\right)\right|\right) \\
& +\rho\left(u\left(r_{t}\right)-p_{t}\right) d c_{t} d p_{t}
\end{aligned}
$$

Thus the objective function is expressed in terms of the current internal state, percept, and control.

The control signal $c_{t}$ is obtained from the SP (logic) layer as a function of the previous state $r_{t-1}$ (cf. fig. 4): $c_{t}=L\left(r_{t-1}\right)$ with the Jacobian $J\left(r_{t-1}\right)=\frac{\partial c_{t}}{\partial r_{t-1}}$. Obviously, the partial derivatives w.r.t. the components in $r_{t-1}$ that are not part of the feedback $f_{t-1}$ vanish, and the Jacobian describes the inputoutput (feedback $f$ / control $c$ ) behavior of the logic layer as a black-box. The Jacobian becomes a factor in the integrand of the objective function by substituting $c_{t}$ and by replacing $\tilde{v}\left(r_{t-1}, u\left(r_{t}\right), p_{t}, r_{t}\right)=v\left(L\left(r_{t-1}\right), \rho\left(u\left(r_{t}\right)-p_{t}\right), r_{t}\right)$ we obtain

$$
\begin{aligned}
\varepsilon(u, \tilde{v})= & \int\left\{\Psi\left(\left|\tilde{v}_{p}\left(r_{t-1}, u\left(r_{t}\right), p_{t}, r_{t}\right)\right|\right)\right. \\
& \left.+\rho\left(u\left(r_{t}\right)-p_{t}\right)\right\} J\left(r_{t-1}\right) d r_{t-1} d p_{t}
\end{aligned}
$$

The advantage of this new formulation is that we can minimize the objective function without explicitly evaluating the logic layer: It is sufficient to know the Jacobian.

The objective function is solved with the standard approach of steepest descent on the Euler-Lagrange equations (where we omit the argument of $\tilde{v}$ )

$$
\begin{aligned}
0=\varepsilon_{u} & =\left\{\Psi^{\prime}\left(\left|\tilde{v}_{p}\right|\right) \frac{\tilde{v}_{p}}{\left|\tilde{v}_{p}\right|} \tilde{v}_{p, u}+\rho^{\prime}\left(u\left(r_{t}\right)-p_{t}\right)\right\} J\left(r_{t-1}\right) \\
0=\varepsilon_{\tilde{v}} & =-\operatorname{div}_{p}\left\{\Psi^{\prime}\left(\left|\tilde{v}_{p}\right|\right) \frac{\tilde{v}_{p}}{\left|\tilde{v}_{p}\right|}\right\} J\left(r_{t-1}\right) \\
& =-\Psi^{\prime \prime}\left(\left|\tilde{v}_{p}\right|\right) \tilde{v}_{p p} J\left(r_{t-1}\right)
\end{aligned}
$$

In order to get the equations for $v$ instead of $\tilde{v}$, we substitute $v$ back. Note that $\tilde{v}_{p}=-v_{\rho} \rho^{\prime}$.

The first equation performs a descent of the two terms of the objective function, i.e., it reduces simultaneously the number of state changes and the prediction error, weighted by the Jacobian. Thus the Jacobian steers the relevance of the error to the state change. The second equation avoids highly curved update functions and thus stabilizes the result by enforcing a local linearization of the update function. Thus, the first term in (12) and the term in (13) have a smoothing effect on $v$ and $u$, whereas the second term in (13) improves fidelity.

As indicated above, we obtain the mappings $u$ and $v$ by gradient descent:

$$
\begin{aligned}
& u_{\text {new }}=u_{\text {old }}-\alpha \varepsilon_{u} \\
& v_{\text {new }}=v_{\text {old }}-\beta \varepsilon_{v}
\end{aligned}
$$

for some suitable step-lengths $\alpha$ and $\beta$ which might also vary with time. Again, terminology is adapted to [4]. If we use a linear model for $u$ giving $u\left(r_{t}\right)=u_{r} r_{t}$, the two updates contain (18) and (19) in [4] as special cases.

Looking at the Euler-Lagrange equation (12) and (13) in more detail, we see that the Jacobian of the next higher level directly influences the updates of the mapping $u$ and $v$ : it might suppress or amplify updates depending on the relevance for the further processing. Updates are in effect logically-weighted. The system so constructed is thus capable of iterative topdown/bottom-up adaptive learning.

This is crucial to the symbolic/sub-symbolic integration proposed in this paper; the multiplicative separability of the Jacobian within the Euler-Lagrange equations implies that we can treat the logic level as a 'black box' that modulates learning on the lower level; we do not therefore need to explicitly combine fuzzy and stochastic algorithms, or give a composite closed-form solution. Instead, we can simply consider only the Jacobian interface. To do this, we shall functionalize the relevant logical components of the declarative reasoning module as follows. This will later enable us to adapt the process for online learning.

\section{Implementing Fuzzy Theorem Proving with a PACF}

In the following, we assume that we have generated a leastfixed-point logical interpretation at time $t$, i.e., $V_{t}^{\log }$. We now wish to relate this to a logic-level virtual system state, $r_{t}^{\log }$ used in the PACF.

Thus, $V_{t}^{\log }=l f p\left(T_{P_{t}}\right)$ where the logic program $P_{t}$ is given here as a tuple separating facts and clauses i.e., $P_{t}=<\wedge_{t^{\prime}}\left\{I s \_\right.$past $\left.\left(t-t^{\prime}\right) \wedge p_{t-t^{\prime}}\right\}, R>$ (such that $R$ is the clause set embodying the Highwaycode and ECOM rules in our application domain). The symbol ' $\wedge t_{t}^{\prime}$ ' indicates the union via conjunction over all $t^{\prime} ; t^{\prime}>0$ ): Is_past is true when its arguments are negative. $P$ is thus an amalgam of the historical perceptual observations and the known a priori rules. The program $P_{t}$ is thus regenerated with each temporal iteration $t=t+1$ (far more complex approaches to temporal logic in the context of uncertainty are possible, cf e.g., [36], however, the current approach is sufficient for our purposes). Note that we write $p_{t}$ as a shorthand for $<p_{t}, v^{\prime} \leftarrow p_{t}>$ i.e. we always consider the atomic percepts $p_{t}$ along with their variables' fuzzy membership allocations.

We may equate $r_{t}^{\log }$ with $P_{t}$ via reasoning as follows. A fact $g$ may be amalgamated with a program $P$ that already embodies the facts $F$ and rules $R$ so as to create a new program $P^{\prime}$ via the relation $P^{\prime}=g \wedge P=<g \wedge F, R>$. The logic-level PACF prediction generation, $\tilde{p}_{t+1}=u^{\log }\left(r_{t}^{\log }, p_{t}\right)$, can thus be modelled on the logic layer by updating $P_{t}$ so as to include the current perceptual observations $p_{t}$ i.e., $P_{t}^{\prime}=I s_{-}$current $(t) \wedge p_{t} \wedge P_{t}$.

A query $v \leftarrow p_{t+1}$ ? directed at the program $P_{t}^{\prime}$ determines the predicted future perceptual state $\tilde{p}_{t+1}$. We denote this $P_{t}^{\prime}\left\{\tilde{p}_{t+1}=v \leftarrow p_{t+1}\right.$ ? $\}$. Hence, the function $u^{\log }$ is defined: $\tilde{p}_{t+1}=u\left(r_{t}^{\log }, p_{t}\right) \equiv P_{t}^{\prime}\left\{\tilde{p}_{t+1}=v \leftarrow p_{t+1} ?\right\}: P_{t}^{\prime}=r_{t}^{\log \wedge}$ $p_{t} \wedge$ Is_current $(t)$

Actions $a$ are passed back to the lower PACF in the form of modified confidences on $p_{t}$ derived from the extrapolation of the globally-consistent model i.e., $a=\tilde{p}_{t}$.

There is no control specified on the topmost logic layer (if this were to exist it would perhaps function as a clause update or rule induction procedure). Thus we can write for the state updater $r_{t}^{\log }=v^{\log }\left(r_{t-1}^{\log }\right)$. This is thus the temporal update function that performs the update equation $r_{t}^{\log }=p_{t} \wedge$ $I s \_p a s t\left(p_{t}\right) \wedge r_{t-1}^{l o g}$ implicit in the above. 
$u^{\log }$ and $v^{\log }$ thus perform the same functions on the logic level as they do on the lower level; they perform the internal state updating and prediction generation in a manner that both minimizes the prediction error (i.e., we accumulate temporal context until the least fixed point $T_{P}(I)=I$ forms a sufficiently consistent world model such that new perceptual input that obeyed the domain code rules does not contradict the fuzzy constraints on the model); they also minimize the internal state updates (i.e., once sufficient temporal context is accumulated, the model only changes in terms of the temporal updating of states, all remaining predication constituting a selfconsistent fixed-point $\left.T_{P}(I)=I\right)^{2}$

The Jacobian 'interface' can thus now be formally written as $\frac{\partial p}{\partial a} \equiv \frac{\partial c}{\partial f}$. However, because of the multiplicative separability of the Jacobian, and corresponding modulation of the EulerLagrange minimization via the declarative fuzzy querying, we are free to treat the logic in its native fuzzy-resolution basedform, without having to derive a closed-form solution for this process. That is, we can maintain the online gradient descent, allowing the logic system to make whatever discrete changes to the update step sizes that accumulated context compels it to do.

\section{The Influence of the Logic-level on the Jacobian: A worked example}

A motivating environment for our work is the area of cognitive driver assistance systems. In this environment, we have a protocol driven domain described by a priori rules (i.e., the Highway Code). The theoretical process above can thus be intuitively related to this practical domain by considering the following (very simplified) example.

Consider the following detector predicates describing key aspects of the current and immediately preceding road configuration:

$$
\begin{aligned}
& \text { obj_pos_left_road }(\text { car_1, current }) \leftarrow[0.7] \\
& \text { obj_pos_road }(\text { car_1, past }) \leftarrow[0.9] \\
& \text { vel_leftwards }(\text { car_1, current }) \leftarrow[0.8]
\end{aligned}
$$

Here, obj indicates object, vel indicates velocity, pos indicates car-relative road position. Thus, the predicate instantiation obj_pos_left_road(car_1,current) refers to a vehicle with label car_1 currently on the left-hand side of the driver's current road. Similarly, obj_pos_road(car_1,past) implies that $c a r \_1$ occupied either side of the road in the preceding interval, since the driver's car entered the junction (i.e., it has not only just, in the current interval, entered the driver's road). vel_leftwards(car_1, current) implies that car_1 has a velocity component to the left of the current road.

Suppose that we also have the following clause determining the driver's (ECOM) intention:

\footnotetext{
driver_intention $($ turn_left, $X) \leftarrow T_{\text {prod }}($

${ }^{2}$ Note that $p$ is sparse with respect to $V^{\log }$ as a whole, as well as being limited to a single temporal slice, and so we would not expect much change in $r^{\log }$ (the 'global' perceptual picture) unless $p$ is a critical predicate (assuming that $p$ is globally consistent). Thus, in general, to a first order of approximation, $r^{\log }$ is modified only in terms of the fuzzy truth values referring to $p$. However, for the cases with only a few predicates, the change in $r^{\log }$ is disproportionately large due to the lack of constraint on $V^{\log }$.
}

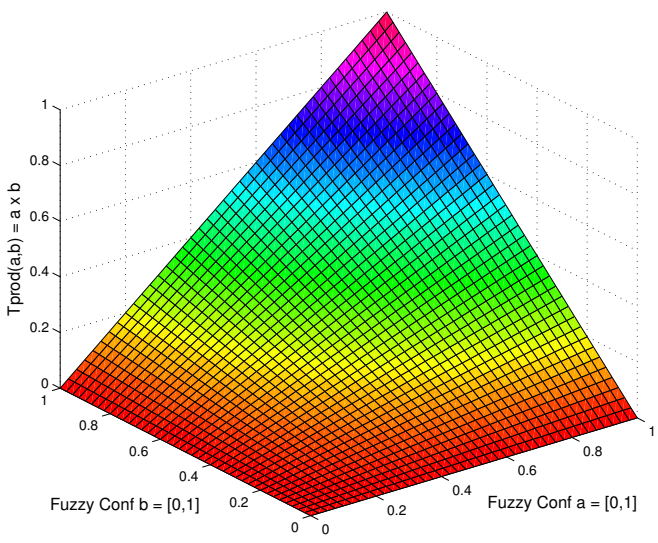

Fig. 5. Graph of the Product T-norm defined over the interval $[0,1]$.

$$
\begin{aligned}
& \text { obj_pos_left_road }(X, \text { current }), \\
& \text { obj_pos_road }(X, \text { past }), \\
& \text { vel_leftwards }(X, \text { current })) .
\end{aligned}
$$

where $X$ varies over car objects.

Single, discrete confidence values define the truth values for each fuzzy predicate (i.e., feature input) - though recall that these are particular instances of a Borel set, rather than standard fuzzy membership values. We apply the simple Product T-norm in this case, $T_{\text {prod }}(x, y)=x \cdot y$ (i.e., an ordinary product of two real numbers). The product T-norm is a strict Archimedean T-norm semantically used for strong conjunction in fuzzy logics.

We are thus provided with three temporal facts,

$$
\begin{aligned}
& \text { obj_pos_left_road (car_1, current), } \\
& \text { obj_pos_road(car_1,past), }
\end{aligned}
$$

and

$$
\text { vel_leftwards (car_1, current) }
$$

whose truth values are the unitary intervals $[0.7,0.7],[0.9,0.9]$, and $[0.8,0.8]$, respectively, as well as a fuzzy clause for the driver_intention predicate, which is defined by an aggregation operator that is a Product T-norm.

Let us consider a fuzzy goal, which seeks to allocate a truth value to the proposition driver_intention(turn_left, $X$ ):

$$
\mu \leftarrow \text { driver_intention(turn_left, } \mathrm{x}) \quad \text { ? }
$$

The first transition in the computation (see equation 6) would be (for initially null $\xi$ ):

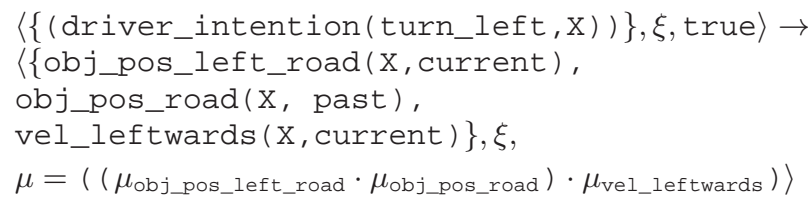

the goal is unified with the clause and the constraint corresponding to Product T-norm is added. The next transition leads to the state:

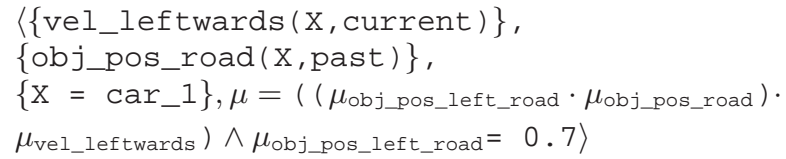


after obj_pos_left_road $(X$, current $)$ is unified with obj_pos_left_road(car_1,current) and the constraint defining the truth value of the fact is added, the computation follows towards the next iteration:

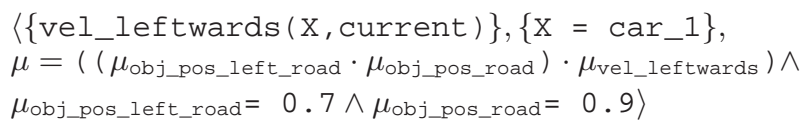

The computation ends with the following iteration:

$$
\begin{aligned}
& \left\langle\{\},\left\{\mathrm{X}=\mathrm{car} \_1\right\}, \mu=\left(\left(\mu_{\text {obj_pos_left_road }} \cdot \mu_{\text {obj_pos_road }}\right) \cdot\right.\right. \\
& \left.\mu_{\text {vel_leftwards }}\right) \wedge \mu_{\text {obj_pos_left_road }}=0.7 \wedge \\
& \left.\mu_{\text {obj_pos_road }}=0.9 \wedge \mu_{\text {vel_leftwards }}=0.8\right\rangle
\end{aligned}
$$

Thus, since:

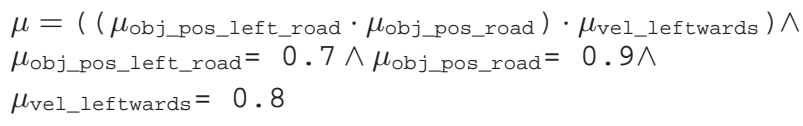

entails $\mu \in[0.50,0.50]$, the answer to the query driver_intention(turn_left,X) is $X=c a r \_l$ with its truth value in the unitary interval $[0.50,0.50]$. Thus given any arbitrary truth values for the fuzzy facts

$$
\text { obj_pos_left_road }(X, T), o b j \_p o s \_r o a d(X, T) \text {, }
$$

and

$$
\text { vel_leftwards }(X, T)
$$

i.e., $[x 1, x 1],[x 2, x 2]$, and $[x 3, x 3]$, respectively, the truth value for the driver intention can be summarized into the following general formula:

$$
\begin{aligned}
& \mu_{\text {intention }}=\left\{T _ { \text { prod } } \left(\mu_{\text {obj_pos_left_road }}, \mu_{\text {obj_pos_driver_road }},\right.\right. \\
& \left.\mu_{\text {vel_leftwards }}\right) \wedge \mu_{\text {obj_pos_left_road }}=\times 1 \wedge \mu_{\text {obj_pos_road }}=x 2 \wedge \\
& \left.\mu_{\text {vel_leftwards }}=x 3\right\}
\end{aligned}
$$

where $\mu_{\text {intention }} \in[0,1]$.

Thus $\mu_{\text {intention }}=x 1 \times x 2 \times x 3$ in this very simplified case.

If we thus treat $\mu_{\text {intention }}$ as the simplified output $a^{\log }$ from the logical level with respect to changes in $p=$ $\left\{\mu_{o b j \_p o s}\right.$ left_road $\left., \mu_{o b j \_p o s \_r o a d}, \mu_{v e l \_l e f t w a r d s}\right\}$, then the Jacobian $\frac{\bar{\partial} p}{\partial a^{\log }}$ becomes:

$$
\frac{\partial p}{\partial a^{\log }}=\left[\begin{array}{lll}
x 2 \times x 3 & x 1 \times x 3 \quad x 1 \times x 2
\end{array}\right]
$$

The Jacobian interfacing of the fuzzy-logic resolution module is similarly applicable in any deductive scenario. We have thus integrated explicit symbolic processing with the stochastic lower-level perception-action learning in the context of a variational Euler-Lagrange framework, and demonstrated that this mapping can be applied in an online context. Crucially, the error of the in-situ P-A mapping exhibits convergent behavior, both for the single layer and also the two layer system, provided that the symbolic processing in the latter provides appropriate priors.

Having thus successfully interfaced the symbolic and subsymbolic levels of the PACF system we hence turn now to an experimental evaluation of the capabilities of the system equipped with this top-down/bottom-up learning capability.

\section{EXPERIMENTAL APPLICATIONS}

We have thus proposed a two-layer PACF module in which on-line learning of top-down symbolic association becomes possible within a deductive logic context. In the following we demonstrate the capability of the composite system within the context of the ECOM model of driver intentionality specified in the Introduction. We thus consider two experimental domains consisting of three scenarios, one quantitative, two qualitative to illustrate these capabilities. Experimental Domain $\mathbf{1}$ is quantitative and seeks to isolate the performance of the two-layer black-box feedback loop. Experimental Domain 2 is qualitative and demonstrates the novel capabilities enabled by online deductive resolution in a P-A mapping context. In particular we demonstrate that the fuzzy logic system is able to update predicates and their confidences in an appropriate fashion when two changing online scenarios are considered.

\section{A. Experiment on Learning with Inter-Layer Feedback}

In Section V, we indicated how learning could be embedded at each level into the layered structure of the overall system, exploiting inter-layer feedback and control during training. The rationale for this approach may be compactly stated in the hypothesis that learning with inter-layer feedback results in improved performance of the trained layer and thus the overall system.

The key result of Section V was thus the characterization of the inter-layer feedback via an appealingly simple modification of single layer learning in terms of a multiplicative factor, see (12) and (13). This feedback factor allows us to verify the hypothesis above in isolation, i.e., without using a particular implementation of the next higher level. Instead, we can assume a certain behavior of that level, choose a suitable learning algorithm for the layer under consideration, and compare the learning performance with and without feedback.

More concretely, we choose the tracking aspect of lowest layer of the driver assistance system for this experiment (also present in the ECOM model given in the Introduction), for which an online version of the method proposed in [37] is used to train the system model of the objects under consideration. At each time step, the method produces a prediction of the object position. The difference between the object position in the current view $p_{t}$ and from the prediction $u\left(r_{t}\right)$ is used to update the system model. The feedback factor is introduced by weighting this error. The experiment itself then consists of comparing the accuracy of the tracker with and without the feedback factor.

In typical circumstances, the second layer in our hierarchy is expected to control the feedback factor in the following way: if the symbolic layer deduces that the current situation is unknown to the system, the tracker would be expected to work poorly, initially, but with the subsequent (re-)learning happening rapidly. On the other hand, if the current situation is known from before, the tracker is supposed to be accurate and discrepancies between tracker prediction and observation are more likely to have originated from detection failures than in the case of an untrained tracker.

Assuming a fixed detection failure rate and an asymptotic convergence of the tracking quality towards optimal performance in the latter case of a novel learning scenario, a suitable model for a well-behaved feedback factor is the decaying 


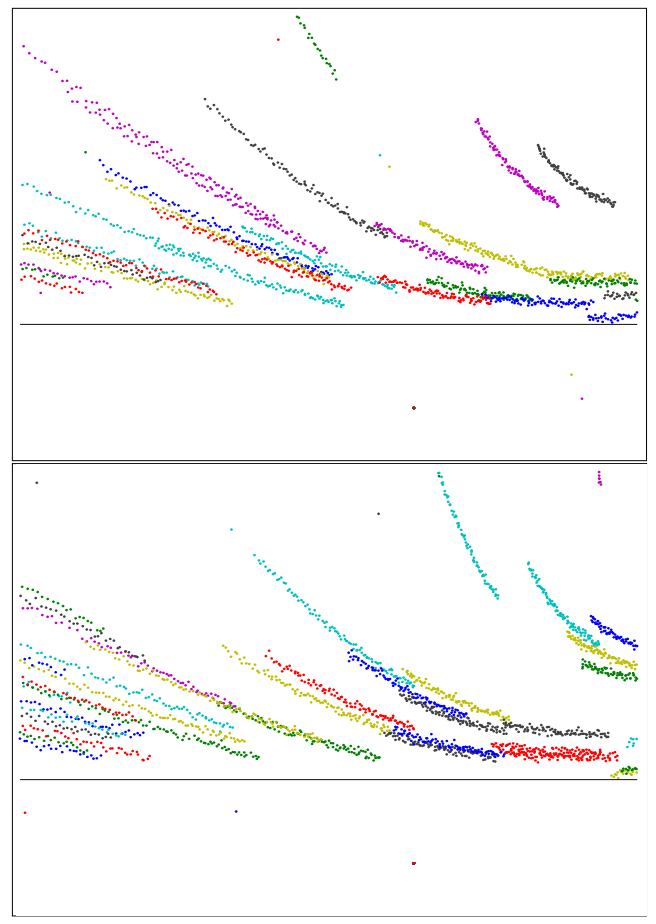

Fig. 6. Two examples for the generated synthetic data. The images show the $3 \mathrm{D}$ point traces in pinhole projection. The black line indicates the horizon.

sequence:

$$
J\left(r_{t}\right)=J_{\min }+\left(J_{\max }-J_{\min }\right) \frac{b}{a t+b}
$$

where $a, b$ are parametric constants. $J_{\min }$ is thus the feedback factor appropriate to the underlying fixed detection failure rate.

In order to perform a quantitative evaluation, we generated synthetic data which is as close to the sequences of traffic sign detections in the driver assistance scenario as possible. We assume a camera on a moving platform that faces ahead. The platform is moving forward with a velocity within some range and the front wheels are allowed to steer within a set angular range. 3D positions are randomly placed in a plane above the ground-plane. These 3D positions are then projected into a pinhole camera model. The resulting 2D points are then distorted by noise and outliers. We thus simulate the situation in which online perception-action mapping at the higher level of the P-A hierarchy performs the function of weighting the error on the mapping of control input (i.e., steering) to relative object velocities, with the higher level anticipating increasing accuracy of the steering/position relation over time. Two examples of such sequences are depicted in Fig. 6. The tracking performance is then evaluated against the groundtruth.

The results of more than 40 runs of our experiments can be summarized as follows: The median error without any feedback is at about $4 \%$ of the image width (the images in Fig. 6 are 500 pixels wide). Introducing a constant feedback factor results in the best case $(J=0.5)$ in a relative accuracy improvement of $3 \%$. If using (17) with $J_{\max }=1, J_{\min }=0.2$, $b=1$, and $a=2$ results in an overall relative accuracy improvement of $10 \%$. This improvement varies very little through the different datasets and minor modifications of parameter.

In order to compare with a standard alternative method, we have also implemented a multiple hypothesis tracking with winner-takes-all association of detections. Points in a frame are always associated to the spatially closest point in the previous frame. Since the motion model is assumed to be unknown, all motion is modeled as noise. Under these assumptions, results are obtained that are significant better than the noise level, but not significantly better than using a constant feedback factor: accuracy is about $3.5 \%$ better than our baseline method without any feedback.

Hence, we conclude that our hypothesis holds and that a suitable top-down feedback improves learning performance significantly. It now remains to demonstrate that higher-level symbol processing can indeed provide an appropriate contextual model for providing feedback.

\section{B. In Situ SP Module Scenarios}

A body of fuzzy clauses is constituted along the lines indicated in Section IV to describe the environment of a driver-assistance system implementing the ECOM model of the Introduction. The outcome of fuzzy deductive resolution is thus a set of detector predicate re-weightings, for which the fuzzy confidences are a measure of the top-down certainty of the detection when the full (spatial/temporal) context is taken into account.

A visual illustration of the maximally self-consistent world model deduced by the logic system is depicted in Fig. 7. This consists in a top-down view of the road junction, with all of the visualizable learned associations and detector predication included as icons. Fuzzy confidences are indicated via alphablending, so that opacity is proportional to confidence (this applies to all entities; road-topology, cars, signs etc).

Higher-level junction traversing ECOM intentions are indicated as red arrows; velocities are depicted as yellow arrows. Where an entity (e.g., a sign) is of unknown type, then the entity is depicted via a question mark icon.

In giving a qualitative illustration of how higher-level symbol processing provides an appropriate contextual model for generating feedback over the Jacobian, we look, in particular at two scenarios capable of illustrating the possibilities enabled by top-down/bottom-up learning:

1) The logical completion (filling-in) of sparse detector predication i.e the supplying of object confidences even in the absence of detections (and detector predication).

2) Adaptive appearance-label/semantic logic association: in particular, the on-line attaching of detector output labels to their functional meanings within the logic system.

The latter scenario is a top-down process intended to exemplify Sloman's notion of symbol tethering [11], wherein an extensible logic system can build on a partially-grounded set of symbols in order to derive a fully grounded symbol set. Thus, ultimately, the system would be able to acquire e.g., novel sign types, by taking novel perceptual clusters identified by the computer vision system and attaching the appropriate functional semantic label to them from the a priori highwaycode rules. 


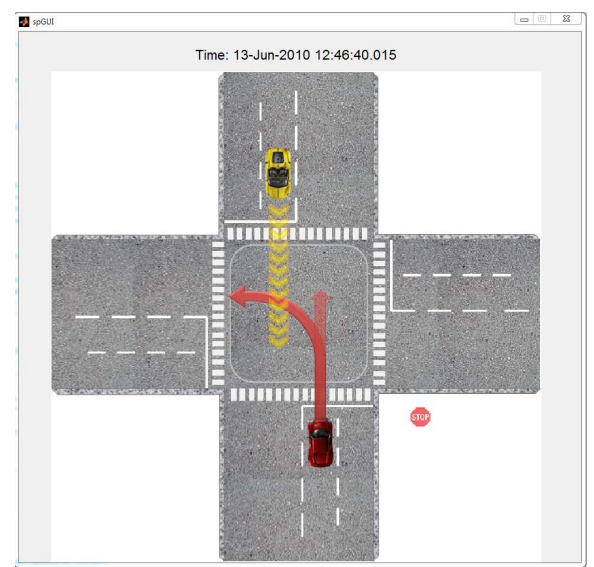

Fig. 7. Typical symbol processing module output, with all visualizable predicates (signs, cars, road topologies) represented by icons with alphablending proportional to fuzzy confidence (expected path trajectories are indicated by yellow arrows, intentions by red arrows).

We look at these scenarios in sequence:

1) Scenario 1: logical completion of sparse detector predication: This scenario represents the default mode of the logic system in the application context, where it attempts to form a stabilized model $r^{\log }$ from the sparse low-level inputs (recall that real input data constitutes only a small fraction of the total set of idealized high-way-code-relevant detectors).

Fig. 8 shows the logic system's accuracy of predicted ECOM intention over time for the most complex junction traverse scenario (i.e., a left turn) -the symbolic output for which is depicted in Fig. 7. In particular, this scenario implicates sign, road and car detectors, as well as gaze and steering input in order to deduce a left-turn intention (Note that turn indicators are blanked out in this scenario, in order to render the deduction non-trivial).

We additionally represent the situation of unstable control inputs by randomly blanking the steering angle control input with a probability of $50 \%$ for each frame (i.e., steering angle is multiplied with a binary 0 or 1 , taken from a Uniform random distribution). Both plots in Fig. 8 indicate that accuracy increases with time as more temporal context is accrued, consistent with our online learning paradigm. As may also be seen, very little accuracy is lost using the noisy data set; the stabilized model $r^{\log }$ is largely impervious to absences of input predication provided that sufficient deductive context can be obtained from elsewhere.

2) Scenario 2: Context Based Semantic Association of Appearance Labels: In the ECOM/highway-code-based logic system, any specific sign detector will instantiate the predicate sign, e.g., sign(stop_sign).

However, in the following illustration of the system's capabilities, we alter the sign detectors so as to instantiate the predicate sign with some unknown, but distinct label, thereby retaining the sign-detection predication associated with sign detection, but depriving the system of any knowledge of sign type i.e., we assert $\operatorname{sign}\left(X_{-} i\right)$ on detecting any particular sign i.

We thus simulate the situation in which a generic sign

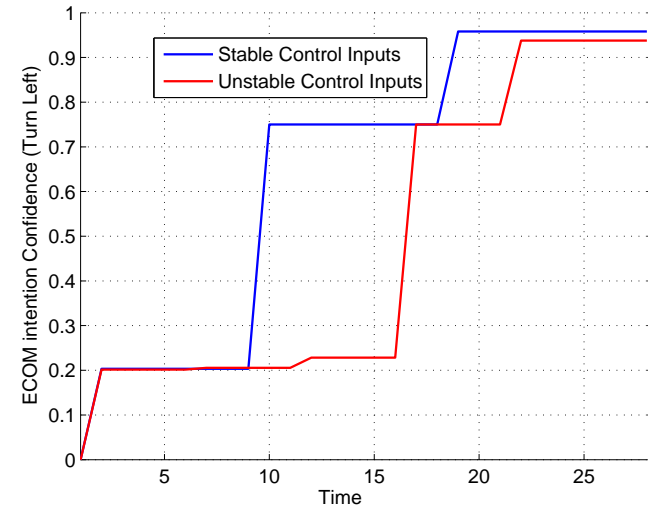

Fig. 8. Confidence of Intention (vert. axis) vs Time (horiz. axis).

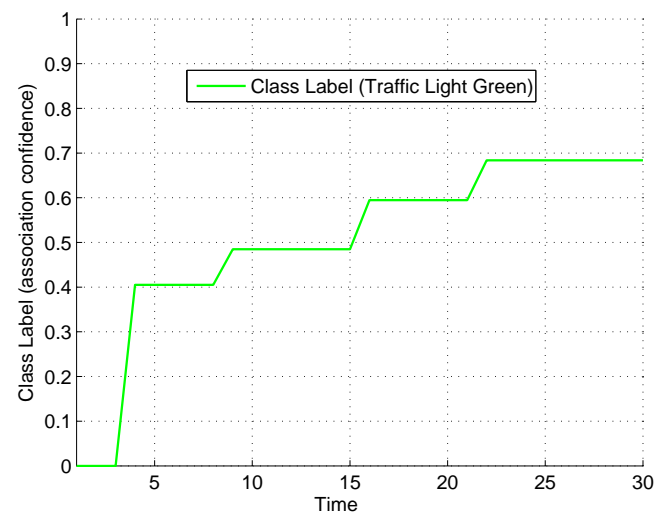

Fig. 9. Confidence in Association of Class ID: 4 with green traffic-light over time.

detector has identified some novel class of sign on the basis of its distinct appearance (i.e., it occurs in a distinct perceptual cluster to the other signs), but which is of unknown type. However the sign type is certain to appear in the high-way code, as contained within the logic system. The goal is then to perform the on-line attachment of the sign detector output labels to their functional meanings within the logic system i.e., carry-out semantic attachment.

We therefore have to associate the driving context (i.e., the behavior of the driver and the other cars with respect to the world model) with a specific a priori sign type. As an additional complicating factor in the following, the specific traffic light states red-light and green-light also have their semantic associations removed (i.e., so that a predicate $\operatorname{light}\left(L_{-} i\right)$ is asserted instead of light(Green) or light(Red) when a light is detected. In the two examples illustrated (Figs. 9 and 10), we express the confidence of symbol attachment in terms of a fuzzy variable plotted over time as a driving sequence (which included several junction traverses) progresses.

In the first example, Fig. 9, we see the progressive confidence in the association of a specific label (Class ID: 4) with green traffic-light. Note, in particular, the increasing confidence with $t$ as data accrues.

In the second example, Fig. 10, an initially wrong asso- 


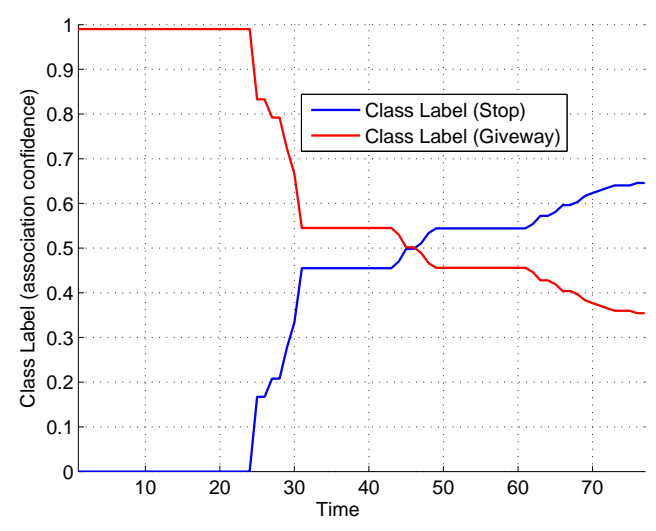

Fig. 10. Confidences in Association of ID: 0 with Stop sign/Give-way sign over time.

ciation of a label (Class ID: 0 ) is made with the semantic class give-way sign, whereas in fact it is a stop sign. We see that the confidence 'flips' to the correct association stop sign when former association becomes untenable after sufficient accrual of contextual data from the other detectors. (All other things being equal, Give-way signs and stop signs are only functionally distinguishable from each other in relation to the behavior of the driver's car with respect to other vehicles traversing the junction).

Predicate confidences are thus adaptively modified on-line by the deductive fuzzy logic system on the basis of the overall spatial/temporal context implied by the other (past and present) detector predicate confidences. Moreover, the feedback supplied by the Symbolic Processing module is consistent with the feedback model assumed by the previous quantitative experiment. By isolating the two levels, we have thus demonstrated that the multiplicative weighting supplied by the SP module across the Jacobian interface is capable of improving the learning convergence rate in accordance with the theoretical predication of Section V.

\section{CONCLUSION AND Future WORK}

We have set out a very general learning framework for online learning in cognitive systems, in which perception-action mapping is carried-out within a symbolic processing context, thereby enabling complex, top-down contextual reasoning to influence the accumulation of low-level competencies. This was achieved by implementing the multi-layer PACF within an Euler-Lagrange framework, and demonstrated that such a system can incorporate first-order logical theorem proving via a Jacobian interface between levels of the subsumptive PA hierarchy. Critically, the multiplicative separability of the Jacobian depends on the use of a fuzzy variant of first-order logical theorem proving.

It was demonstrated experimentally in the context of an intelligent driver assistance system that the resulting framework achieves significantly greater performance than perceptionaction learning alone, as well as permitting novel forms of context-dependant P-A mapping. We have thus achieved online integration of symbolic and sub-symbolic processing in combined Perception-Action learning. In practical applications this composite system may be employed to address the classical issues of entity resolution and semantic/appearancelabel association, where there exist strong deductive contextual constraints. We also note that domain rules need not be specified a priori; fuzzy rule discovery is also possible [38]. It can can thus be employed in open-ended domains, where no a priori knowledge of the domain rules is available, requiring the use of rule induction to link Perceptions to Actions (which could proceed along the lines of [39]).

Our method can be thus applied in almost any domain of embodied autonomous cognition for perception-action learning (this includes a wide class of problems, including e.g., SLAM robotics). It can also be used to model human intentional activity in protocol driven environment. We finally note that the presence of a deductive component within a system that naturally grounds symbols within a perceptionaction hierarchy means that the current system fulfils Sloman's criterion of a symbol tethering system [11].

\section{ACKNOWLEDGMENT}

The research leading to these results has received funding from the European Community's Seventh Framework Programme (FP7/2007-2013) under grant agreements no. 215078 (DIPLECS) and 247947 (GARNICS). We also acknowledge support from the Swedish Foundation for Strategic Research for the project CUAS (Collaborative Unmanned Aerial Systems), and from UK EPSRC grant EP/F069626/1 (ACASVA).

\section{REFERENCES}

[1] Brooks, R.A.: Intelligence without representation. Artif. Intell. 47 (1991) 139-159

[2] Granlund, G.H.: The complexity of vision. Signal Processing 74 (1999) 101-126

[3] Felsberg, M., Wiklund, J., Granlund, G.: Exploratory learning structures in artificial cognitive systems. Image and Vision Computing 27 (2009) 1671-1687

[4] Rao, R.P.N.: An optimal estimation approach to visual perception and learning. Vision Research 39 (1999) 1963-1989

[5] Fidler, S., Leonardis, A.: Towards scalable representations of object categories: Learning a hierarchy of parts. In: 2007 IEEE Computer Society Conference on Computer Vision and Pattern Recognition (CVPR 2007), 18-23 June 2007, Minneapolis, IEEE Computer Society (2007)

[6] Shevchenko, M., Windridge, D., Kittler, J.: A linear-complexity reparameterisation strategy for the hierarchical bootstrapping of capabilities within perception-action architectures. Image Vision Comput. 27 (2009) 1702-1714

[7] Harnad, S.: The symbol grounding problem. Phys. D 42 (1990) 335-346

[8] Kelley, T.D.: Symbolic and sub-symbolic representations in computational models of human cognition what can be learned from biology? Theory \& Psychology 13(6) (2003) 847-860

[9] Kruger, N.: Three dilemmas of signal- and symbol-based representations in computer vision. In Gregorio, M., Maio, V., Frucci, M., Musio, C., eds.: Brain, Vision, and Artificial Intelligence. Lecture Notes in Computer Science, Springer (2005) 167 - 176

[10] Schlemmer, M., Prankl, J., Vincze, M.: Vision for situated robot companions; fusing top-down knowledge and bottom-up data. In: AFRICON'09. (2009)

[11] Schlemmer, M.J., Vincze, M.: Theoretic foundations of situating cognitive vision in robots and cognitive systems. Journal of Experimental \& Theoretical Artificial Intelligence 24 (2012)

[12] Hollnagel, E., Woods, D.D. In: Joint Cognitive Systems: Foundations of Cognitive Systems Engineering. CRC Press, Taylor \& Francis Group (2005) 149-154

[13] Rao, R.P.N., Ballard, D.H.: Predictive coding in the visual cortex: a functional interpretation of some extra-classical receptive-field effects. Nature Neuroscience 2 (1999) 79-87 
[14] Felsberg, M., Granlund, G.: Fusing dynamic percepts and symbols in cognitive systems. In: Int. Conference on Cognitive Systems. (2008)

[15] Schon, T.B., Eidehall, A., Gustafsson, F.: Lane departure detection for improved road geometry estimation. In: Proc. of the IEEE Intelligent Vehicles Symposium Tokyo, Japan, June 2006. (2006) 546-551

[16] Neisser, U.: Cognition and Reality: Principles and Implications of Cognitive Psychology. W. H. Freeman, San Francisco (1976)

[17] Felsberg, M., Larsson, F.: Learning Bayesian tracking for motion estimation. In: International Workshop on Machine Learning for Visionbased Motion Analysis. (2008)

[18] Richardson, M., Domingos, P.: Markov logic networks. Mach. Learn. 62 (2006) 107-136

[19] Jain, D., Kirchlechner, B., Beetz, M.: Extending markov logic to model probability distributions in relational domains. In: KI '07: Proc. of the 30th annual German conference on Advances in Artificial Intelligence, Berlin, Heidelberg, Springer-Verlag (2007) 129-143

[20] Kok, S., Domingos, P.: Learning markov logic network structure via hypergraph lifting. In: In Proceedings of the 26th International Conference on Machine Learning (ICML-09). (2009)

[21] Klir, G.J., Yuan, B.: Fuzzy Sets and Fuzzy Logic: Theory and Applications. 1st edn. Prentice Hall PTR, NJ, USA (1995)

[22] Mendel, J.: Fuzzy logic systems for engineering: a tutorial. Proceedings of the IEEE 83 (1995) $345-377$

[23] Qiao, J., Yang, N., Gao, J.: Two-stage fuzzy logic controller for signalized intersection. Systems, Man and Cybernetics, Part A: Systems and Humans, IEEE Transactions on 41 (2011) 178 -184

[24] Chen, S.M.: A fuzzy reasoning approach for rule-based systems based on fuzzy logics. Systems, Man, and Cybernetics, Part B: Cybernetics, IEEE Transactions on 26 (1996) $769-778$

[25] Shapiro, E.Y.: Logic programs with uncertainties: a tool for implementing rule-based systems. In: IJCAI'83: Proceedings of the Eighth international joint conference on Artificial intelligence, San Francisco, CA, USA, Morgan Kaufmann Publishers Inc. (1983) 529-532

[26] Vaucheret, C., Guadarrama, S., Mu noz-Hernández, S.: Fuzzy prolog: A simple general implementation using clp(r). In: Proceedings of the 18th International Conference on Logic Programming. (2002) 469

[27] Dickerson, J., Kosko, B.: Fuzzy function approximation with ellipsoidal rules. Systems, Man, and Cybernetics, Part B: Cybernetics, IEEE Transactions on 26 (1996) $542-560$

[28] Jenei, S.: On the convex combination of left-continuous t-norms. aequationes mathematicae 72 (2006) 47-59

[29] Alsina, C., Frank, M., Schweizer, B.: Problems on associative functions, Aequationes Mathematicae 66 (2003) 128-140

[30] Detyniecki, M., Yager, R.R., Bouchon-meunier, B.: Reducing t-norms and augmenting t-conorms (2002)

[31] Lee, C.: Fuzzy logic in control systems: fuzzy logic controller. i. Systems, Man and Cybernetics, IEEE Trans. on 20 (1990) $404-418$

[32] Cheong, F., Lai, R.: Constraining the optimization of a fuzzy logic controller using an enhanced genetic algorithm. IEEE Transactions on Systems, Man, and Cybernetics, Part B 30 (2000) 31-46

[33] Chen, S.M., Chang, Y.C.: Weighted fuzzy rule interpolation based on ga-based weight-learning techniques. Fuzzy Systems, IEEE Transactions on 19 (2011) $729-744$

[34] Jaffar, J., Maher, M.J.: Constraint logic programming: a survey. The Journal of Logic Programming 19-20 (1994) 503 - 581

[35] Munoz-Hernandez, S., Sari Wiguna, W.: Fuzzy cognitive layer in robocupsoccer. In: IFSA '07: Proc. of the 12th international Fuzzy Systems Association world congress on Foundations of Fuzzy Logic and Soft Computing, Springer-Verlag (2007) 635-645

[36] Moon, S., Lee, K., Lee, D.: Fuzzy branching temporal logic. Systems, Man, and Cybernetics, Part B: Cybernetics, IEEE Transactions on 34 (2004) $1045-1055$

[37] Felsberg, M., Larsson, F.: Learning higher-order Markov models for object tracking in image sequences. In: ISVC. Volume 5876 of LNCS. (2009) 184-195

[38] Lee, W.J., Lee, S.J.: Discovery of fuzzy temporal association rules. Systems, Man, and Cybernetics, Part B: Cybernetics, IEEE Transactions on 34 (2004) $2330-2342$

[39] Windridge, D., Shevchenko, M., Kittler, J.: Open-ended inference of relational representations in the cospal perception-action architecture. In: International Cognitive Vision Workshop. (2007)

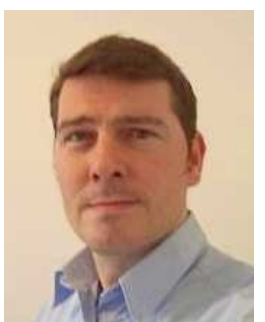

David Windridge (BSc (Hons) 1993, MSc 1995, $\mathrm{PhD}$ 1999) is a Senior Research Fellow in the University of Surrey, having research interests in Pattern Recognition and Cognitive Systems, as well as a former research interest in Observational Cosmology. His grant portfolio includes a range of EPSRC and EU cognitive systems projects (most recently EPSRC ACASVA and EU FP7 DIPLECS), as well as a number of industrial and academic pattern recognition projects. His early career commenced in industrial electro-acoustic research, which was followed by a research-based M.Sc in Radio-Astronomy, leading to a Ph.D in Cosmology (Univ. of Bristol, UK). This led to his current position at the CVSSP (Univ. of Surrey, UK), since when his remit expanded to include statistical pattern-recognition and cognitive systems. He has authored more than 60 peer-reviewed publications.

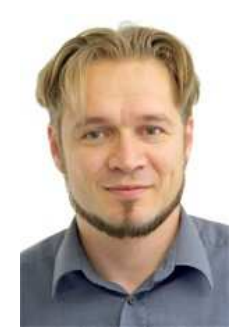

Michael Felsberg PhD degree (2002) in engineering from University of Kiel. Since 2008 full professor and head of CVL. Research: signal processing methods for image analysis, computer vision, and machine learning. More than 80 reviewed conference papers, journal articles, and book contributions. Awards of the German Pattern Recognition Society (DAGM) 2000, 2004, and 2005 (Olympus award), of the Swedish Society for Automated Image Analysis (SSBA) 2007 and 2010, and at Fusion 2011 (honourable mention). Coordinator of EU projects COSPAL and DIPLECS. Associate editor for the Journal of Real-Time Image Processing, area chair ICPR, and general co-chair DAGM 2011.

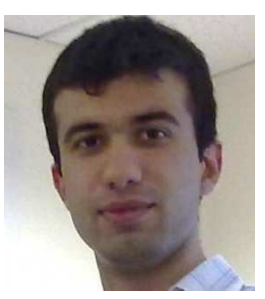

Affan Shaukat received the B.Eng. degree (with First-class honours) in Electronic Engineering in 2008 from the University of Surrey, Guildford, United Kingdom. He is currently working toward the Ph.D. degree in Electronic Engineering at the Centre for Vision, Speech and Signal Processing, University of Surrey, Guildford, United Kingdom. He was actively involved in the EU (FP-7 ICT) project DIPLECS over the course of his research degree. He has authored and co-authored scientific articles in various conference and workshop venues. His research interests include topics in cognitive computation, machine learning, artificial intelligence and pattern recognition. 